Revista Eletrônica de Direito Processual - REDP. Volume 16. Julho a dezembro de 2015

Periódico Semestral da Pós-Graduação Stricto Sensu em Direito Processual da UERJ

Patrono: José Carlos Barbosa Moreira. ISSN 1982-7636. pp. 93-111

http://www.e-publicacoes.uerj.br/index.php/redp/index

\title{
LA VICINANZA DELLA PROVA ${ }^{1}$
}

\section{THE “PROOF'S NEIGHBOURHOOD” PRINCIPLE}

Chiara Besso Marcheis

Professore Ordinario di Diritto Processuale Civile nell'Università degli Studi di Torino, Italia.

chiara.bessomarcheis@unito.it

SOMMARIO: 1 . Premessa: la regola dell'onere della prova. - 2. La vicinanza della prova ovvero la riscoperta di un tema classico. - 3. L'applicazione del criterio della vicinanza della prova da parte della giurisprudenza. - 4. Il fenomeno non è limitato all'ordinamento italiano (in particolare, gli ordinamenti ibero-americani). - 5. Disponibilità e vicinanza della prova nel processo amministrativo. -6 . Una proposta di lettura del fenomeno: vicinanza della prova e accesso alla prova (un cenno alla disclosure in materia di proprietà industriale). - 7. Conclusione: dall'onere alla collaborazione delle parti nella raccolta delle prove.

1. - La regola dell'onere della prova è presente nella generalità degli ordinamenti ${ }^{2} \mathrm{e}$, sostanzialmente basata sul brocardo onus probandi incubit ei qui dicit, è volta a risolvere il problema della incertezza circa i fatti rilevanti per la decisione addossando il rischio del mancato convincimento del giudice alla parte che ha allegato il fatto. L'idea, alla base della regola, è che è colui che vuole modificare in suo favore lo status quo a dover dare la prova della verità delle sue pretese e quindi a soccombere, lasciando così immutato lo stato esistente delle cose, ove in tale compito fallisca ${ }^{3}$.

Nell'ordinamento italiano l'onere della prova è codificato in via generale all'art. 2697 c.c., che distribuisce il rischio sulla base della partizione dei fatti in costitutivi ovvero modificativi,

\footnotetext{
${ }^{1}$ Artigo recebido em 29/10/2015, sob dispensa de revisão.

${ }^{2} \mathrm{Si}$ veda al riguardo il quadro tracciato da Taruffo, Onere della prova, in Dig. disc. priv., sez. civ., XIII, 1995, 65 ss.

${ }^{3}$ Lévy-Bruhl, La preuve judiciaire, Paris, 1964, 39 s.; Taruffo, La semplice verità. Il giudice e la ricostruzione dei fatti, Bari, 2009, 227 s.; Bergeaud, Le droit à la preuve, Paris, 2010, 24 s.
} 
Revista Eletrônica de Direito Processual - REDP. Volume 16. Julho a dezembro de 2015

Periódico Semestral da Pós-Graduação Stricto Sensu em Direito Processual da UERJ

Patrono: José Carlos Barbosa Moreira. ISSN 1982-7636. pp. 93-111

http://www.e-publicacoes.uerj.br/index.php/redp/index

estintivi e impeditivi. La disposizione, apparentemente semplice e chiara ${ }^{4}$, in realtà presenta, come sanno gli operatori pratici, innumerevoli complicazioni applicative.

Essa viene intesa come rinvio alla fattispecie sostanziale sottostante alle pretese fatte valere dalle parti, rinvio ritenuto esclusivo ovvero filtrato dalla posizione processuale rivestita da ciascuna parte ${ }^{5}$. Prescindiamo dalle, numerose, ipotesi in cui il legislatore direttamente interviene sulla ripartizione degli oneri probatori attraverso la tecnica delle presunzioni ${ }^{6}$. È illusorio pensare che la norma sostanziale dia un criterio vincolante certo per l'attribuzione del rischio della prova. Da un lato nella norma sostanziale non troviamo fatti costitutivi accanto a fatti modificativi, estintivi o impeditivi della fattispecie, ma solo fatti assunti come elementi della fattispecie ${ }^{7}$ e dall'altro lato, in ogni caso, quelli previsti dal legislatore inevitabilmente sono "abbozzi" semplificati dei casi reali ${ }^{8}$.

La determinazione dell'onere finisce così per essere operata dal giudice sulla base di criteri che completano e qualificano la fattispecie legale adeguandola al caso concreto, quali l'apparenza, l'interesse, la normalità, il carattere negativo del fatto da dimostrare9 .

Al proposito si segnala, negli ultimi anni, il richiamo prevalente da parte della giurisprudenza italiana al criterio della vicinanza (o riferibilità, prossimità, facilità) della prova, tanto che la giurisprudenza, di legittimità e di merito, è giunta a parlare di «dogma» della vicinanza della prova ${ }^{10}$.

A una riflessione sul significato del criterio della vicinanza della prova è dedicato il presente contributo, che partendo da un inquadramento della nozione è volto, dopo aver esaminato l'utilizzo che di essa viene fatto, anche in altri ordinamenti e tipi di processo, a suggerire una lettura della sua accresciuta importanza.

\footnotetext{
4 "Pura formula didattica" la definisce Cordero, Guida alla procedura penale, Torino, 1986, 371.

${ }^{5}$ Il riferimento è alle due più importanti ricostruzioni dottrinarie della regola ex art. 2697, ossia quella di Verde, L'onere della prova nel processo civile, Camerino, 1974, e di Micheli, L'onere della prova [1941], rist., Padova, 1966.

${ }^{6}$ In argomento, per tutti, Sacco, Presunzione, natura costitutiva o impeditiva del fatto, onere della prova, in Riv. dir. civ., 1957, 399 ss.

${ }^{7}$ L'osservazione è di Benvenuti, L'istruzione nel processo amministrativo Padova, 1953, 400, nota 53.

${ }^{8}$ Così Verde, L'onere della prova nel processo civile, cit., 465.

${ }^{9}$ Ancora Verde, op. loc. cit.

${ }^{10}$ Così Cass., 20 febbraio 2006, n. 3651 (in relazione a un'azione di risarcimento del danno, in conseguenza di un incidente stradale, proposta contro l'ANAS sulla base dell'art. 2051 c.c.); di "principio dogmatico di c.d. vicinanza alla prova" parla pure App. Torino, 28 marzo 2007 (la pronunzia può essere letta in www.dejure.it).
} 
Revista Eletrônica de Direito Processual - REDP. Volume 16. Julho a dezembro de 2015

Periódico Semestral da Pós-Graduação Stricto Sensu em Direito Processual da UERJ

Patrono: José Carlos Barbosa Moreira. ISSN 1982-7636. pp. 93-111

http://www.e-publicacoes.uerj.br/index.php/redp/index

2. - L'identificazione tra parte onerata e parte che, vicina alla prova, può agevolmente fornire la dimostrazione del fatto indipendentemente dalla sua allegazione, non è nuova.

È a Bentham cui dobbiamo il fondamento teorico del tema. Nella sua monumentale opera sulle prove, il filosofo inglese contrappone infatti l'imposizione dell'onere della prova imperniata sull'allegazione del fatto, propria di quella che egli chiama la procedura tecnica, all'imposizione dell'onere invece alla parte che sia in grado di dimostrare il fatto con il minore inconveniente (ove inconveniente, specifica Bentham, significa ritardo, vessazione e spese), propria della procedura naturale. Se la seconda impostazione è caratterizzata dalla semplicità, l'applicazione dell'aforisma tecnico dell'onere della prova "si presenta intralciata da nodi che è più facile tagliare che sciogliere" ${ }^{11}$.

L'impostazione di Bentham ha trovato un'eco nella dottrina italiana in Pescatore, che ha costruito la sua teoria delle prove attraverso il confronto diretto con il pensiero del filosofo inglese $\mathrm{e}^{12}$.

Pescatore rifiuta l'impostazione radicale di Bentham e afferma la validità della regola "che in giudicio dopo aver allegato è d'uopo provare" ${ }^{13}$. Tuttavia, precisa l'autore, "certe condizioni di fatto portano con sé stesse tal quale probabilità naturale e quando a questa generale verosimiglianza s'aggiunga la difficoltà estrema che incorrerebbe l'attore a doverne dare una prova più rigorosa, la facilità della prova contraria per parte del convenuto e la necessità di ovviare a più gravi perturbazioni nella pratica della vita civile, la legge e la giurisprudenza tradizionale, che ne tiene le veci, introducono una presunzione in favore dell'attore ossia una eccezione alla regola generale che gli imporrebbe per tutte le condizioni di fatto il carico della prova".

Pescatore, a differenza di Bentham, vede quindi il criterio della vicinanza alla prova porsi non come alternativo al sistema della allegazione, ma come suo temperamento.

11 Bentham, An Introductory View of the Rationale of Evidence, in The Works of Jeremy Bentham, a cura di Bowring, Edinburgh, 1843, vol. 6, cap. XXVIII (Of the burthen of proof: on whom shall it lie?).

${ }^{12} \mathrm{Si}$ veda al riguardo Besso, Pescatore, voce del Dizionario biografico dei giuristi italiani, II, Bologna, 2013, 1553 S.

${ }_{13}$ Pescatore, Logica del diritto. Frammenti di dottrina e di giurisprudenza, II ed., Torino, 1883, I, 95 ss., in particolare 95 e 106. 
Revista Eletrônica de Direito Processual - REDP. Volume 16. Julho a dezembro de 2015

Periódico Semestral da Pós-Graduação Stricto Sensu em Direito Processual da UERJ

Patrono: José Carlos Barbosa Moreira. ISSN 1982-7636. pp. 93-111

http://www.e-publicacoes.uerj.br/index.php/redp/index

È questa la posizione della dottrina, e della giurisprudenza, successiva. La vicinanza della

prova è uno, e non il più importante (centrale, pensiamo al pensiero di Chiovenda ${ }^{14}$, è infatti ritenuto quello della normalità del fatto da dimostrare), dei criteri utilizzati dal giudice per attribuire l'onere della prova, impostazione che permane con il passaggio dalla formulazione dell'art. $1312^{15}$ - presente nel codice civile del 1865 - a quella dell'art. 2697.

Così Verde ${ }^{16}$ - e siamo nel 1974 - nell'individuare i criteri sottostanti alla determinazione dei rischi probatori e analizzarne l'uso da parte dei giudici, esamina, separatamente, i criteri dell'interesse, della normalità e della specificità per poi affermare che gli altri criteri (tra i quali, appunto, la vicinanza alla prova) sono meno invocati dai giudici e menzionare tre sole pronunzie che sulla base della vicinanza alla prova hanno distribuito tra le parti l'onere di dimostrare ${ }^{17}$.

3. - Decisiva, per il passaggio della vicinanza della prova da criterio raramente invocato a principio invece centrale del sistema, è stata la pronunzia n. 13533/2001 delle sezioni unite della Corte di cassazione.

Chiamate a comporre il contrasto, esistente in giurisprudenza, circa l'applicazione della regola dell'onere della prova nelle cause in cui viene chiesta la risoluzione per inadempimento del contratto, le sezioni unite hanno scelto l'orientamento minoritario, inaugurato da una pronunzia del $1996^{18}$. La terza sezione della Corte aveva infatti osservato che la riferibilità della

\footnotetext{
${ }^{14}$ Istituzioni di diritto processuale civile, II, Napoli, 1934, 316 ss.

${ }^{15} \mathrm{La}$ disposizione, che poneva una regola generale in materia di obbligazioni ("chi domanda l'esecuzione di un'obbligazione deve provarla e chi pretende esserne stato liberato deve dal canto suo provare il pagamento o il fatto che ha prodotto l'estinzione della sua obbligazione"), è una traduzione di quella ancora oggi dettata dall'art. 1315 del code civil francese: «Celui qui réclame l'exécution d'une obligation doit la prouver. Réciproquement, celui qui se prétend libéré doit justifier le paiement ou le fait qui a produit l'extinction de son obligation ».

${ }^{16}$ Verde, L'onere della prova nel processo civile, 465 ss., in particolare 473 e 475.

${ }^{17}$ Rispettivamente, Cass. n. 1720/1968 in materia di lavoro (era stata chiesta da parte del lavoratore l'indennità per lavori nocivi), Cass. n. 2368/1970, in materia di regolare convocazione dell'assemblea da parte dell'amministratore di condominio (vi era stata l'impugnazione della deliberazione dell'assemblea condominiale), App. Milano, 29 settembre 1972 (in Foro it., 1974, I, 1251), circa la prova da parte della società che la valutazione degli immobili contenuta nel bilancio corrisponda al vero. La situazione non muta nel decennio successivo: si veda, ad esempio, una pronuncia del Tribunale di Roma degli anni 1980, ove la vicinanza della prova è uno dei criteri menzionati dal giudice (la "prova dell'adeguato apporto del concedente, quale elemento che lo esenta dalla conversione in affitto del contratto di mezzadria, incombe sul predetto sulla base dei principi della normalità, specificità, della vicinanza alla prova e della non negatività di questa", Trib. Roma, 6 novembre 1987 in Riv. dir. agr., 1988, 241).

${ }_{18}$ Cass., 7 febbraio 1996, n. 973, poi ripresa da Cass., 27 marzo 1998, n. 3232, e da Cass., 15 ottobre 1999 , n. 11629.
} 
Revista Eletrônica de Direito Processual - REDP. Volume 16. Julho a dezembro de 2015

Periódico Semestral da Pós-Graduação Stricto Sensu em Direito Processual da UERJ

Patrono: José Carlos Barbosa Moreira. ISSN 1982-7636. pp. 93-111

http://www.e-publicacoes.uerj.br/index.php/redp/index

prova è criterio al quale si ispira l'art. 2697, distinguendo tra fatti costitutivi, modificativi ed estintivi, cosicché è conforme alla norma che la prova dell'adempimento, fatto estintivo del diritto per la cui tutela agisce il creditore (sia che agisca in via diretta che per il risarcimento) spetti al debitore, convenuto in giudizio, che dovrà dare la prova diretta e positiva di un fatto riferibile alla sua sfera di azione.

Le sezioni unite riprendono il ragionamento della sezione semplice e - in un caso ove l'attore, che inizialmente aveva proposto una domanda di adempimento, poi mutata, ai sensi dell'art. 1453, comma 2 c.c., in domanda di risoluzione e richiesta di risarcimento del danno, aveva dimostrato l'avvenuta conclusione del contratto, ma si era poi limitato ad affermare, senza darne prova, che l'obbligazione non era stata adempiuta - hanno imposto l'onere della prova dell'adempimento al convenuto.

L'importanza della pronunzia sta non tanto (o meglio, non solo) nell'imposizione dell'onere di dimostrare l'adempimento al convenuto anche in caso di domanda di risoluzione del contratto - che a tale risultato si potrebbe arrivare guardando alla sola fattispecie normativa ${ }^{19}$ - quanto nelle argomentazioni offerte dalla Corte che superano la prospettiva della fattispecie sostanziale.

L'operare della regola dell'onere della prova va infatti ricondotto - dicono le sezioni unite - non ad astratti criteri di costruzione della fattispecie, ma a criteri di ragionevolezza ${ }^{20}$ : visto che il presupposto dell'azione di adempimento è lo stesso delle azioni di risoluzione e di risarcimento del danno, ossia l'inadempimento, allora se per la prima azione è sufficiente l'allegazione dello stesso, è ragionevole che l'allegazione sia sufficiente anche per le altre due azioni. D'altro canto - e qui il ragionamento delle sezioni unite assume una valenza generale che trascende l'ipotesi dell'azione di risoluzione del contratto - dimostrare da parte del debitore di aver adempiuto è più facile che provare il fatto negativo inadempimento da parte del creditore; l'esigenza di non rendere eccessivamente difficile l'esercizio del diritto di difesa impone quindi

\footnotetext{
${ }^{19}$ L'art. 1453, infatti, prevedendo alternativamente la condanna all'adempimento o la risoluzione del contratto parrebbe subordinarli al medesimo fatto. Cfr. però i rilievi di Verde (L'onere della prova, cit., 424 ss.), che, pur affermando che la posizione assunta dalla parte nel processo non gioca alcun ruolo, finisce per aderire alla tesi (sostenuta anzitutto da Micheli, L'onere della prova, cit., 438 ss.) che vuole l'adempimento dimostrato dall'attore che agisce per la risoluzione.

20 "L'eccesso di distinzioni di tipo concettuale e formale - dice la Corte - è fonte di difficoltà per gli operatori diritto".
} 
Revista Eletrônica de Direito Processual - REDP. Volume 16. Julho a dezembro de 2015

Periódico Semestral da Pós-Graduação Stricto Sensu em Direito Processual da UERJ

Patrono: José Carlos Barbosa Moreira. ISSN 1982-7636. pp. 93-111

http://www.e-publicacoes.uerj.br/index.php/redp/index

che sia la parte che è vicina alla prova quella sulla quale va addossato l'onere di produrre in giudizio gli elementi utili per la decisione del giudice e quella sulla quale grava il rischio della mancata prova.

Il dictum delle sezioni unite è stato ben presto applicato a numerose ipotesi, ulteriori rispetto a quelle delle cause di risoluzione per inadempimento. Mi limito ad alcuni esempi.

Anzitutto, la soluzione scelta per l'inadempimento viene estesa - seguendo un obiter dictum espresso dalle sezioni unite nella stessa pronuncia n. 13533/2001 ${ }^{21}$ - all'inesatto adempimento, in settori classici come quello della compravendita di beni ${ }^{22}$ e in settori "nuovi" come quello della responsabilità del medico e della struttura sanitaria, ove sono numerosissime le pronunzie ove si afferma che «il paziente che agisce in giudizio deducendo l'inesatto adempimento dell'obbligazione sanitaria deve provare il contratto e allegare l'inadempimento del sanitario, restando a carico del debitore provare l'esatto adempimento» ${ }^{23}$.

Ricordo ancora il settore delle cause di lavoro - ove le sezioni unite della Corte hanno utilizzato il criterio della vicinanza per addossare la prova del "requisito occupazionale" al

\footnotetext{
21 "Le richiamate esigenze di omogeneità del regime probatorio - hanno affermato le sezioni unite - inducono a estendere anche all'ipotesi dell'inesatto inadempimento il principio della sufficienza dell'allegazione dell'inesattezza dell'adempimento, gravando anche in tale eventualità sul debitore l'onere di dimostrare l'avvenuto esatto adempimento".

${ }^{22}$ Cfr., ad esempio, Cass., 2 settembre 2013, n. 20110, ove, in un caso in cui il compratore aveva chiesto la risoluzione del contratto di fornitura di collante per legno con condanna della parte convenuta al risarcimento del danno, la Corte ha affermato che "in tema di inadempimento del contratto di compravendita, è sufficiente che il compratore alleghi l'inesatto adempimento, ovvero denunci la presenza di vizi che rendano la cosa inidonea all'uso al quale è destinata o ne diminuiscano in modo apprezzabile il valore, mentre è a carico del venditore, quale debitore di un'obbligazione di risultato ed in forza del principio della riferibilità o vicinanza della prova, l'onere di dimostrare, anche attraverso presunzioni, di aver consegnato una cosa conforme alle caratteristiche del tipo ordinariamente prodotto, ovvero la regolarità del processo di fabbricazione o di realizzazione del bene".

${ }^{23}$ Così Trib. Bari, 8 luglio 2009, n. 2300, nonché tra le decisioni della Corte di cassazione da ultimo si segnalano Cass., 20 ottobre 2014, n. 22222, e Cass., 27 agosto 2014, n. 18304.
} 
Revista Eletrônica de Direito Processual - REDP. Volume 16. Julho a dezembro de 2015

Periódico Semestral da Pós-Graduação Stricto Sensu em Direito Processual da UERJ

Patrono: José Carlos Barbosa Moreira. ISSN 1982-7636. pp. 93-111

http://www.e-publicacoes.uerj.br/index.php/redp/index

datore di lavoro convenuto dal lavoratore che fa valere l'illegittimità del licenziamento ${ }^{24}-$, la materia dei diritti dei consumatori ${ }^{25}$, quella bancaria $^{26}$ e quella societaria ${ }^{27}$.

Al di là delle azioni e degli ambiti in cui viene applicato, quale ruolo gioca il criterio e qual è il suo rapporto con la disposizione di cui all'art. 2697?

Se la vicinanza alla prova è criterio che addossa l'onere della rappresentazione del fatto in modo indipendente rispetto alla sua introduzione, diversa è la tecnica ermeneutica invocata dalla giurisprudenza nelle pronunzie esaminate.

In talune decisioni - come nella sentenza delle sezioni unite n. 13533/2001 - la vicinanza/distanza della prova è utilizzata per identificare tra $\mathrm{i}$ fatti allegati quelli che sono costitutivi della pretesa e che quindi devono essere provati dall'attore, così ponendosi all'interno dell'art. 2697, che viene interpretato come se dicesse: chi vuol fare valere un diritto in giudizio deve provare i fatti costitutivi della stessa, ossia quelli la cui prova è nella sua disponibilità, mentre quelli che non è in grado di provare sono estintivi (o modificativi e impeditivi) e devono essere rappresentati dalla controparte.

\footnotetext{
${ }^{24}$ Cass., sez. un., 10 gennaio 2006, n. 141, in Foro it., 2006, I 704. Ancora in materia di lavoro si veda la pronuncia della Corte di cassazione n. 20484/2008, ove in una causa in cui il dipendente di una banca chiedeva il riconoscimento del premio di produttività, la Corte ha affermato che la "ripartizione dell'onere della prova tra lavoratore, titolare del credito, e datore di lavoro, deve tenere conto, oltre che della partizione della fattispecie sostanziale tra fatti costitutivi e fatti estintivi od impeditivi del diritto, anche del principio (..) della riferibilità o vicinanza o disponibilità dei mezzi di prova".

${ }^{25}$ Cfr. Cass., 28 maggio 2014, n. 11904, ove un consumatore aveva agito in giudizio contro la compagnia assicuratrice chiedendone la condanna al risarcimento del danno causato dalla violazione delle norme sulla concorrenza così come accertata dall'Autorità garante per la concorrenza e il mercato: "il consumatore assolve l'onere della prova a suo carico con la produzione della documentazione dell'AGCM e del contratto da lui stipulato. Sull'impresa sanzionata grava, anche alla stregua del principio di vicinanza della prova (..), l'onere di vincere la presunzione, dimostrando che l'ammontare del premio non sia stato determinato anche dalla condotta anticoncorrenziale, ma soltanto da fattori ad essa estranei".

${ }^{26} \mathrm{Da}$ ultimo si veda Trib. Ancona, ord. 28 gennaio 2015, per il quale "nel giudizio di ripetizione dell'indebito, se non sono stati depositati gli estratti conto fin dall'inizio del rapporto e il saldo contabile risulti negativo per il correntista, deve assumersi, quale base del riconteggio, un saldo di partenza uguale a zero, in quanto il principio dell'onere della prova di cui all'art. 2697 deve essere adeguatamente temperato avendo riguardo al principio della vicinanza alla fonte della prova che le sezioni unite (..) hanno elevato a criterio principe nella ripartizione dell'onere stesso". In dottrina cfr. Dolmetta-Malvagna, Vicinanza della prova in materia di contenzioso bancario, in Riv. dir. bancario, 2014, n. 6, 1 ss.

${ }^{27}$ Cfr. Cass., 5 febbraio 2015, n. 2156, ove, in relazione all'azione ex art. 2449 c.c., la Corte sostiene che non spetta alla parte che agisce in giudizio dimostrare che gli atti degli amministratori sono espressione della normale attività d'impresa e non hanno finalità liquidatoria: "questa interpretazione, oltre che con il principio di vicinanza della prova avente ad oggetto fatti a diretta conoscenza della società e dei suoi amministratori è coerente con la loro natura di fatti impeditivi o modificativi del diritto che il creditore ha azionato allegando e provando i relativi fatti costitutivi".
} 
Revista Eletrônica de Direito Processual - REDP. Volume 16. Julho a dezembro de 2015

Periódico Semestral da Pós-Graduação Stricto Sensu em Direito Processual da UERJ

Patrono: José Carlos Barbosa Moreira. ISSN 1982-7636. pp. 93-111

http://www.e-publicacoes.uerj.br/index.php/redp/index

In altre pronunce, invece, il criterio si pone all'esterno dell'art. 2697 e costituisce un temperamento della partizione tra fatti costitutivi e fatti estintivi, modificativi od impeditivi del diritto: il fatto, quindi, mantiene la sua qualificazione, ma il criterio esterno alla norma fa sì che della dimostrazione dello stesso la parte sia esonerata.

Altre decisioni, ancora, richiamano - come già indicava Pescatore ${ }^{28}$ - lo schema della presunzione: così, in materia di responsabilità medica e sanitaria, viene affermato che «la sussistenza del nesso eziologico tra la patologia e il pregiudizio si deve presumere allorché sia impossibile accertare e valutare altri ipotetici fattori causali proprio in conseguenza della lacunosa compilazione della cartella clinica ${ }^{29}$. In altre, infine, il richiamo alla vicinanza della prova serve per giustificare l'applicazione della disposizione che pone una presunzione legale relativa in favore dell'attore: così, circa l'art. 2051 c.c., la Corte di cassazione ${ }^{30}$ afferma che «la presunzione posta dalla norma è da intendersi quale finzione che, mediante un'eccezione al principio generale posto dall'art. 2697, determina una distribuzione dell'onere della prova diversa rispetto a quella valevole in tema di illecito civile per la regola generale di cui all'art. 2043 c.c., al fine di favorire il danneggiato».

4. - Prima di tentare una lettura del fenomeno, ritengo utile allargare l'orizzonte dell'indagine.

L'attenzione, nella attribuzione del rischio della mancata dimostrazione del fatto, al criterio della vicinanza alla prova è infatti fenomeno non circoscritto all'ordinamento italiano.

Il pensiero va anzitutto agli ordinamenti ibero-americani ${ }^{31}$, ove da tempo si sottolinea la rigidità della regola tradizionale che addossa il rischio della mancata dimostrazione alla parte

\footnotetext{
${ }^{28}$ Supra, paragrafo 2.

${ }^{29}$ Cass., n. 10060/2010. Sulla qualificazione giuridica della cartella clinica cfr. Occorsio, Cartella clinica $e$ vicinanza della prova, in Riv. dir. civ., 2013, 1249 ss. Lo strumento della presunzione è richiamato, sempre in materia sanitaria, da Cass., 7 ottobre 2014, n. 21083, ove in relazione alla domanda proposta contro il ministero della salute per ottenere il risarcimento del danno per aver contratto l'epatite $\mathrm{C}$ a seguito di una emotrasfusione: “accertata l'esistenza della patologia da virus (..) in soggetto emotrasfuso è corretta l'ulteriore deduzione per la quale, in assenza di altri fattori alternativi, le omissioni di controllo e vigilanza addebitabili al ministero sono state causa dell'insorgenza della malattia (..) e sussisteva perciò in capo al ministero, anche in considerazione del principio della vicinanza della prova, l'onere di vincere la presunzione suddetta e di provare di aver adottato le condotte necessarie per evitare la contagiosità".

${ }^{30}$ Cass., 20 febbraio 2006, n. 3651.

${ }^{31}$ Il criterio è richiamato anche in Francia ove si parla di "parte che appare la più idonea a dare la prova" (cfr. Cadiet-Normand-Amarani Mekki, Théorie generale du procés, Paris, 2013, n. 255). Nei Paesi Bassi, poi, l'art. 150
} 
Revista Eletrônica de Direito Processual - REDP. Volume 16. Julho a dezembro de 2015

Periódico Semestral da Pós-Graduação Stricto Sensu em Direito Processual da UERJ

Patrono: José Carlos Barbosa Moreira. ISSN 1982-7636. pp. 93-111

http://www.e-publicacoes.uerj.br/index.php/redp/index

che allega il fatto e si parla invece di distribuzione dinamica dell'onere della prova ${ }^{32}$, ove l'onere

è ripartito dal giudice sulla base appunto della disponibilità o facilità che la parte ha nel dimostrare i fatti del processo.

Il criterio della disponibilidad y facilidad probatoria, da un paio di decenni oggetto di applicazione da parte della giurisprudenza, particolarmente in settori "sensibili" caratterizzati dalla asimmetria tra le posizioni delle parti (quali la tutela del diritto del consumatore e del malato), ha trovato espressa codificazione in Spagna e, quest'anno, pure in Brasile.

Il nuovo codice di rito spagnolo, ponendosi sulla scia di una serie di pronunzie del Tribunal Supremo, che hanno affermato che «la norma che distribuisce l'onere della prova non risponde a un principio inflessibile, ma va adattata al caso concreto, a seconda della natura dei fatti affermati o negati e della disponibilità o facilità di prova di ciascuna parte ${ }^{33}$, ha previsto all'ultimo comma dell'art. 217 - ove è disciplinato in modo dettagliato l'onere della prova ${ }^{34}$ che nell'applicare i precedenti commi, ed in particolare il secondo e il terzo, che riproducono il

del codice di rito, dopo aver affermato che la parte ha l'onere di provare il fatto le cui conseguenze giuridiche fa valere, fa salva l'ipotesi in cui "esigenze di ragionevolezza e di equità pongano un diverso onere della prova", equità che viene appunto identificata nella facilità probatoria della parte (si veda al riguardo Laenens, Rapporto nazionale al XII Congresso dell'Associazione internazionale di diritto processuale, Città del Messico, 2003, ripreso dalla relazione di Cadiet, Culture et administration judiciaire de la preuve, n. 24).

32 È all'argentino Peyrano (di cui cfr. La regla de la carga de la prueba enfocada come norma de clausura del sistema, in Civil Procedure Review, 2010, n. 3, 95 ss.) che viene attribuita la paternità dell'espressione "distribuzione dinamica dell'onere della prova".

33 Tribunal Supremo, 9 marzo 1991, nonché le altre pronunzie menzionate da Pazos Méndez, Los criterios de facilidad y disponibilidad probatoria en el proceso civil, in Abel Lluch-Picó I Junoy (a cura di), Objecto y carga de la prueba civil, Barcelona, 2007, 79.

${ }^{34}$ L'art. 217 della Ley de Enunciamento Civil del 2000, originariamente di sette commi, si articola oggi, dopo l'abrogazione del quinto, in sei commi. Il comma 1 dispone che, ove il giudice in sede di decisione della causa ritenga dubbia l'esistenza di un fatto rilevante per la decisione, deciderà circa le rispettive pretese delle parti a seconda che spettasse all'una o all'altra l'onere di provare il fatto rimasto incerto. Il comma 2 afferma che spetta all'attore l'onere di provare la certezza dei fatti su cui la sua pretesa si basa, secondo le norme giuridiche ad essa applicabili e l'effetto giuridico ad essa corrispondente. Secondo il comma 3 spetta al convenuto l'onere di provare i fatti che, secondo le norme giuridiche ad essi applicabili, impediscono, modificano o estinguono l'efficacia giuridica dei fatti cui si riferisce il precedente comma. Il comma 4 attribuisce al convenuto l'onere della prova in materia di concorrenza sleale e pubblicità illecita (come faceva l'abrogato comma 5 per le azioni in materia di discriminazione, oggi sostituito dall'art. 13 della legge n. 3/2007 per l'effettiva uguaglianza di donne e uomini). Il comma 5 precisa che le regole dettate ai precedenti commi trovano applicazione a meno che una diversa distribuzione dell'onere sia prevista da una norma speciale. Il comma 6, infine, è quello riportato in testo, che appunto codifica il criterio della facilità probatoria. 
Revista Eletrônica de Direito Processual - REDP. Volume 16. Julho a dezembro de 2015

Periódico Semestral da Pós-Graduação Stricto Sensu em Direito Processual da UERJ

Patrono: José Carlos Barbosa Moreira. ISSN 1982-7636. pp. 93-111

http://www.e-publicacoes.uerj.br/index.php/redp/index

nostro art. 2697, il giudice «deve considerare la disponibilità e facilità della prova di ciascuna delle parti della lite» ${ }^{35}$.

L'introduzione del criterio $^{36}$, considerato uno dei profili più rilevanti della nuova disciplina $^{37}$, è stata accolta con cautela dalla dottrina. Si sottolinea la necessità di evitare un utilizzo eccessivo del criterio da parte del giudice che possa portare a una situazione di incertezza giuridica per le parti e se ne afferma così il carattere residuale e sussidiario ${ }^{38}$. Dal punto di vista dello strumento tecnico, si osserva che l'applicazione del criterio non determina un'inversione dell'onere, ma l'eliminazione del fatto la cui dimostrazione risulta troppo difficile per la parte dall'elenco dei fatti che essa deve provare.

Quanto al Brasile, anche qui preceduta da applicazioni giurisprudenziali, la facilità della prova è oggi prevista, in via generale, dal recentissimo nuovo codice di procedura civile ${ }^{39}$. All'art. 373, dopo un $1^{\circ}$ comma che ricalca, mediante l'attribuzione dei fatti costitutivi all'attore e dei fatti impeditivi, modificativi o estintivi al convenuto, il nostro art. 2697, si dispone che quando le peculiarità della causa rendono impossibile o eccessivamente difficile l'adempimento dell'onere ovvero sia più facile provare il fatto contrario, il giudice può attribuire l'onere della

\footnotetext{
${ }^{35}$ Si vedano Pazos Méndez, Los criterios de facilidad y disponibilidad probatoria, cit., 77 ss., e Fachal Noguer, Las reglas de la carga de la prueba en la responsabilidad civil médica: cuestiones polémicas, nel medesimo lavoro curato da Abel Lluch e Picó I Junoy, supra citato, 210 ss.

${ }^{36} \mathrm{Il}$ precetto, assente nel progetto di codice del 1998, è stato inserito successivamente dopo che a favore della sua introduzione si era espresso il consiglio generale del potere giudiziario. Un precedente viene individuato nell'art. 129 del codice processuale civile modello dell'Istituto ibero-americano (si veda il Testo dell'avanprogetto, Montevideo, 1988). L'articolo - intitolato onere della prova - dopo una prima parte che richiama il nostro art. 2697 ("è onere di chi fa valere una pretesa dimostrarne i fatti costitutivi, mentre chi alla pretesa si oppone ha l'onere di provare i fatti modificativi, impeditivi o estintivi della stessa"), dispone che la distribuzione dell'onere della prova non è di ostacolo all'iniziativa probatoria del giudice né alla valutazione, secondo le regole della sana crítica, delle omissioni o deficienze della produzione di prove.

${ }^{37}$ Cfr. Pazos Méndez, op. cit., 79.

${ }^{38} \mathrm{La}$ facilità della prova costituisce invece la regola-base del codice modello dei processi collettivi ibero-americano (Código Modelo de Procesos Colectivos, Caracas, ottobre 2004). All'art. 12, dopo aver disposto l'ammissibilità di qualsiasi prova, purché ottenuta in modo lecito, la legge-modello prevede al comma 2 che l'onere della prova spetta alla parte che detiene le conoscenze scientifiche, tecniche o le informazioni specifiche circa i fatti ovvero che può più facilmente provare $\mathrm{i}$ fatti, e poi ai commi successivi prevede correttivi alla regola-base (mediante l'attribuzione al giudice del potere di dare gli ordini necessari per supplire la mancanza e ottenere le prove indispensabili per la pronuncia di merito). Per un commento della disposizione cfr. Bujosa Vadell, La posición del juez en el código modelo de procesos colectivos para Iberoamérica, in www.ambito-juridico.com.br.

${ }^{39}$ Legge n. 13.105 del 16 marzo 2015; il codice entrerà in vigore nel 2016.
} 
Revista Eletrônica de Direito Processual - REDP. Volume 16. Julho a dezembro de 2015

Periódico Semestral da Pós-Graduação Stricto Sensu em Direito Processual da UERJ

Patrono: José Carlos Barbosa Moreira. ISSN 1982-7636. pp. 93-111

http://www.e-publicacoes.uerj.br/index.php/redp/index

prova in modo diverso, a condizione che ciò faccia con una decisione motivata, dando alla parte

la possibilità di soddisfare l'onere ad essa assegnato ${ }^{40}$.

5. Anche nell'ordinamento italiano il criterio della disponibilità probatoria ha trovato espresso riconoscimento da parte del legislatore ${ }^{41}$. Mi riferisco al processo amministrativo.

In questo processo, strutturalmente caratterizzato da squilibrio informativo tra le parti, l'istruttoria è imperniata, secondo la felice formula di Benvenuti ${ }^{42}$, sul principio dispositivo acquisitivo $^{43}$ : processo di parti, cui è comunque addossato l'onere dell'allegazione dei fatti, esso tradizionalmente presenta cospicui poteri di iniziativa istruttoria d'ufficio da parte del giudice ${ }^{44}$.

La previsione del potere del giudice di intervenire per colmare le lacune dell'istruzione, unita alla mancanza di una espressa regola di decisione del fatto incerto equivalente all'art. 2697, ha portato la dottrina a ritenere che la parte che ha adempiuto all'onere di introdurre $i$ fatti possa essere sollevata dall'onere di rappresentarli ${ }^{45}$. La giurisprudenza ha così affermato che sul ricorrente grava un onere consistente «nel fornire non già la prova piena dei fatti su cui si fondi la domanda, stante la disponibilità in mano alla pubblica amministrazione della documentazione probatoria (c.d. riferibilità o vicinanza o disponibilità del mezzo) bensì di allegare il principio

\footnotetext{
${ }^{40}$ Per un commento della proposta contenuta nel progetto del codice cfr. Marinoni-Mitidiero, O projeto do CPC, São Paulo, 2010, 102 ss., i quali sottolineano - come abbiamo visto in testo fanno gli autori spagnoli - la differenza tra dinamica e inversione dell'onere della prova, in quanto il primo fenomeno, a differenza del secondo, comporta una attribuzione ex novo dell'onere in relazione alle circostanze della singola causa. Per il collegamento tra distribuzione dinamica dell'onere della prova e giusto processo cfr. dos Santos Rodrigues, A effectividade do proceso e a distribuição do ônus da prova, in Revista Electrônica de Direito Processual, XII, 545 ss.

${ }^{41}$ Cfr. T.A.R. Catanzaro (Calabria), 8 maggio 2013, n. 539, per il quale "il principio della c.d. vicinanza della prova ha trovato un addentellato normativo nel codice del processo amministrativo all'art. 64".

${ }^{42}$ Benvenuti, L'istruzione nel processo amministrativo, sopra citata. Al riguardo cfr., per tutti, Gallo C.E., La prova nel processo amministrativo, Milano, 1994, 37 ss.

${ }^{43}$ Cons. stato, adunanza plenaria, 23 marzo 2011, n. 3. Per un generale giudizio, critico, circa l'istruttoria del processo amministrativo cfr. Chizzini, I poteri istruttori, in Sassani e Villata, Il processo davanti al giudice amministrativo, Torino, 2001, 233 ss.

${ }^{44}$ Pensiamo all'art. 44 del testo unico sul Consiglio di stato (R.D. n. 1054/1924): "se la sezione, a cui è stato rimesso il ricorso riconosce che l'istruzione dell'affare è incompleta, o che i fatti affermati nell'atto o provvedimento impugnato sono in contraddizione coi documenti, può richiedere all'amministrazione interessata nuovi schiarimenti o documenti”.

${ }^{45}$ Il riferimento è, ancora, a Benvenuti (op. cit., 442 s., 449 s.), per il quale l'art. 44 t.u. cons. stato, insieme al potere acquisitivo, accordava al giudice il potere di influire sulla ripartizione dell'onere della prova attribuendolo con carattere di novità alla parte che con più facilità è in grado di superare l'incertezza. Non molto diversa è la conclusione cui giungeva Micheli (op. cit., 282 s.): il giudice amministrativo ripartisce l'incertezza, fissando caso per caso la regola di giudizio in base alla quale decidere, regola che "gli è in genere dettata da criteri di esperienza, primo fra i quali la maggior vicinanza di una parte alla prova".
} 
Revista Eletrônica de Direito Processual - REDP. Volume 16. Julho a dezembro de 2015

Periódico Semestral da Pós-Graduação Stricto Sensu em Direito Processual da UERJ

Patrono: José Carlos Barbosa Moreira. ISSN 1982-7636. pp. 93-111

http://www.e-publicacoes.uerj.br/index.php/redp/index

di prova cioè indicare tali fatti suscettibili di trovare o meno conferma mediante acquisizione a cura del giudice di quella documentazione ${ }^{46}$.

Una disciplina dell'onere della prova si è avuta nel 2010, con il codice del processo amministrativo. L'articolo da considerare è il 64 del codice ${ }^{47}$, rubricato «disponibilità, onere e valutazione della prova».

Se il comma 3 dispone circa il potere d'ufficio del giudice («il giudice amministrativo può disporre, anche d'ufficio, l'acquisizione di informazioni e documenti utili ai fini del decidere che siano nella disponibilità della pubblica amministrazione»), i commi secondo e quarto ripropongono il testo di disposizioni del processo civile in punto valutazione delle prove e obbligo per il giudice di porre alla base della sua decisione le prove proposte dalle parti ${ }^{48}$.

È il primo comma quello che ci interessa: spetta alle parti «l'onere di fornire gli elementi di prova che siano nella loro disponibilità riguardanti i fatti posti a fondamento delle domande e delle eccezioni». La norma pone l'accento sulla disponibilità, e quindi sulla vicinanza, della prova invece che sulla allegazione del fatto, così allontanandosi dalla regola fissata dall'art. 2697.

La dottrina amministrativistica ha focalizzato la propria attenzione sui rapporti tra onere probatorio imposto alle parti ed esercizio del potere istruttorio del giudice, interrogandosi così sulla permanenza o meno del principio acquisitivo ${ }^{49}$, dando in certo senso come scontato l'onere di ciascuna parte di fornire le prove che rientrano nella propria disponibilità, indipendentemente dalla circostanza che queste siano favorevoli ovvero sfavorevoli rispetto alla pretesa da essa vantata.

\footnotetext{
${ }^{46}$ Così, tra le ultime pronunzie precedenti il codice del 2010, Cons. stato, 16 luglio 2007, n. 3875.

${ }^{47}$ D.lgs. 2 luglio 2010, n. 104, c.d. codice del processo amministrativo.

${ }^{48} \mathrm{Il}$ comma 2 ("salvi i casi previsti dalla legge, il giudice deve porre a fondamento della decisione le prove proposte dalle parti nonché i fatti non specificatamente contestati dalle parti costituite") è una trascrizione dell'art. 115, comma 1, c.p.c.; il comma 4 ("il giudice deve valutare le prove secondo il suo prudente apprezzamento e può desumere argomenti di prova dal comportamento tenuto dalle parti nel corso del processo") riproduce parte dell'art. 116 c.p.c.

${ }^{49}$ È positiva la risposta in genere data dagli autori, almeno per le ipotesi in cui nel processo viene sindacato il potere della pubblica amministrazione (si veda, per tutti, Gallo C.E., I poteri istruttori del giudice amministrativo - Report annuale 2011, in www.ius-publicum.com) anche se non manca chi invece sostiene (ad esempio, Ballesini, La disciplina dell'onere della prova nel codice del processo amministrativo, in Rass. dell'Avvocatura dello Stato, 2011, 3, 139 ss.) che, con il codice, si è avuto il superamento della tesi di Benvenuti, così che il processo amministrativo sarebbe oggi basato sul solo principio dispositivo (tesi che mi pare difficile condividere se si tiene presente il comma 3 dell'art. 64, riportato in testo).
} 
Revista Eletrônica de Direito Processual - REDP. Volume 16. Julho a dezembro de 2015

Periódico Semestral da Pós-Graduação Stricto Sensu em Direito Processual da UERJ

Patrono: José Carlos Barbosa Moreira. ISSN 1982-7636. pp. 93-111

http://www.e-publicacoes.uerj.br/index.php/redp/index

È invece questo il profilo che interessa lo studioso del processo civile, ove la vigenza di

un simile principio è tutt'altro che scontata ${ }^{50}$. Interesse che a mio avviso è tanto più rilevante quando si considera che l'art. 64 vale per la generalità dei processi che si svolgono davanti al giudice amministrativo, quindi non soltanto quelli volti a sindacare la legittimità dell'atto della pubblica amministrazione, ma anche quelli di risarcimento del danno consequenziale e quelli, di giurisdizione esclusiva, aventi ad oggetto la tutela di diritti soggettivi.

Certo, è legittimo chiedersi se l'onere imposto dal comma 1 dell'art. 64 sia regola solo probatoria o che invece svolga pure il ruolo di distribuire il rischio della mancata prova (addossandolo quindi alla parte nella cui disponibilità la prova si trova).

Rimanendo nel solco della proposta interpretativa di Benvenuti, la regola di cui al comma 1 dell'art. 64 è sia regola probatoria che regola di giudizio, anche se occorre ricordare che questi subordina l'addossamento del rischio della prova all'ordine istruttorio del giudice, che attribuisce così ex novo alla parte in grado di assolverlo il rischio del mancato convincimento in ordine al fatto allegato, ma non dimostrato ${ }^{51}$.

6. - Il brevissimo e parziale excursus che abbiamo svolto ci dice alcune cose.

Il problema della ripartizione del rischio della mancata prova è problema avvertito in tutti gli ordinamenti e trova una trattazione che - pur nella diversità di accento sull'esercizio di poteri discrezionali da parte del giudice ${ }^{52}$ - presenta aspetti comuni.

\footnotetext{
${ }^{50}$ Diversa pare l'opinione degli amministrativisti: si veda Saitta (Onere della prova e poteri istruttori del giudice amministrativo dopo la codificazione, in www.giustamm.it, 2012, n. 7, 13 s.) per il quale il criterio della vicinanza alla prova è principio regolatore della distribuzione dell'onere da sempre utilizzato nel processo civile.

${ }^{51}$ Benvenuti, op. cit., 449 s.

${ }^{52}$ Negli ordinamenti di common law vengono tradizionalmente riconosciuti ampi poteri discrezionali al giudice nella ripartizione dell'onere della prova (in tal senso, riassuntivamente, Taruffo, La semplice verità, cit., 232). La differenza non va, però, a mio avviso sopravvalutata. Se infatti leggiamo trattazioni classiche del tema da parte di giuristi americani (pensiamo a quelle di Wigmore, A Treatise on the Anglo-american System of Evidence, III ed., vol. 9, Boston, 1940, 272 ss., e di Fleming, Burdens of Proof, in 47 Virginia Law Review, 1961, 58 ss.) e le confrontiamo con quelle poste in essere da Micheli e, soprattutto, da Verde (rispettivamente, L'onere della prova e L'onere della prova nel processo civile, più volte menzionate) la distanza appare più di linguaggio che di sostanza. Così Wigmore afferma che, anzitutto, la distribuzione dell'onere della prova avviene sulla base della legge sostanziale, che fissa i gruppi di dati che, alla base delle relazioni giuridiche, costituiscono i diritti e i doveri che fondano le pretese delle parti, e poi della legge del processo, che suddivide i gruppi di dati in sottogruppi assegnando l'uno o l'altro a questa o a quella parte, per infine passare a considerare i criteri di equità (equità che Wigmore collega alla facilità di accesso della parte alla prova) che devono essere considerati quando si attribuisce l'onere della prova nel caso specifico.
} 
Revista Eletrônica de Direito Processual - REDP. Volume 16. Julho a dezembro de 2015

Periódico Semestral da Pós-Graduação Stricto Sensu em Direito Processual da UERJ

Patrono: José Carlos Barbosa Moreira. ISSN 1982-7636. pp. 93-111

http://www.e-publicacoes.uerj.br/index.php/redp/index

È però negli ordinamenti che appartengono alla tradizione di civil law che il tema della

vicinanza della prova appare negli ultimi anni particolarmente sentito, tanto da essersi tradotto, in alcuni di essi, in espressa regola normativa di applicazione generale ${ }^{53}$.

La ragione sta, a mio avviso, nella minore capacità di questi ordinamenti di garantire l'accesso alla prova rispetto a quelli che appartengono alla famiglia del common law. In questi ultimi - come ben sappiamo - è presente una fase del processo, la discovery, che è finalizzata alla "scoperta" delle prove ${ }^{54}$. L'estensione della discovery varia: massima negli Stati Uniti (ove è possibile prima del trial chiedere alla controparte o a un terzo, attraverso i mezzi di discovery, tutte le informazioni che possono ragionevolmente portare a prove ammissibili) è più limitata in Inghilterra ${ }^{55}$, ove, comunque, ciascuna parte, a pena di sanzioni molto severe, ha l'obbligo di far conoscere alla controparte sia i documenti ad essa favorevoli che quelli ad essa invece sfavorevoli o che comunque provano la pretesa avversaria ${ }^{56}$.

La discovery è invece estranea agli ordinamenti di civil law ${ }^{57}$, storicamente imperniati sul principio espresso dal brocardo nemo tenetur edere contra se. È vero che negli ultimi decenni il principio ha iniziato a essere scalfito e anche nell'Europa continentale limitati obblighi di produzione di documenti sono stati introdotti.

Pensiamo all'ordinamento italiano, ove è previsto - unica significativa novità in materia di prove del codice del 1940 - il potere del giudice di ordinare, su istanza di parte, la produzione di documenti detenuti dalla controparte o dal terzo. L'obbligo è però, oltre che debolmente sanzionato, rigorosamente circoscritto: i documenti di cui si chiede l'esibizione devono essere specificamente individuati e deve essere fornita la prova che la controparte li detiene ${ }^{58}$.

\footnotetext{
${ }^{53}$ Si veda Lagarde, D'une vérité l'autre, in Gazette du Palais, Doctrine, 21-22 luglio 2010, 2024, il quale osserva come i giudici di common law siano poco interessati alle regole di attribuzione del burden of proof, che appare oggi questione marginale del processo.

${ }^{54} \mathrm{Cfr}$. al riguardo il nostro lavoro La prova prima del processo, Torino, 2004, 66 ss.

55 In Inghilterra la discovery ha infatti tradizionalmente avuto ad oggetto solo i documenti detenuti dalla controparte. Le Civil Procedure Rules (CPR), entrate in vigore nel 1999, se da un lato hanno limitato lo scambio di documenti tra le parti (la c.d. standard discovery, ribattezzata disclosure), dall'altro lato hanno contemplato la disclosure contro il terzo e codificato lo scambio, prima del trial, delle deposizioni dei testimoni (cfr., per tutti, ZUCKERMAN, Civil Procedure. Principles of Practice, London, 2013, 718 ss.).

${ }^{56} \mathrm{Si}$ veda in particolare la Rule 31.6 delle CPR.

${ }^{57}$ Sulle origini storiche dell'istituto vedere il nostro La discovery anglo-americana: un insospettato trapianto dal processo romano-canonico, in Aequitas sive deus, II, Torino, 2011, 1193 ss.

${ }^{58}$ Cfr. l'art. 94 disp. att. del nostro codice di rito.
} 
Revista Eletrônica de Direito Processual - REDP. Volume 16. Julho a dezembro de 2015

Periódico Semestral da Pós-Graduação Stricto Sensu em Direito Processual da UERJ

Patrono: José Carlos Barbosa Moreira. ISSN 1982-7636. pp. 93-111

http://www.e-publicacoes.uerj.br/index.php/redp/index

Né una vera discovery (nonostante il termine venga evocato in dottrina $\mathrm{e}$ in giurisprudenza) è presente in settori particolari quale quello della proprietà industriale ${ }^{59}$. L'art. 121 del codice ${ }^{60}$, intitolato "ripartizione dell'onere della prova", dopo aver dettato al comma 1 una regola di distribuzione del carico della prova $^{61}$, al comma 2 stabilisce una disciplina dell'esibizione, speciale rispetto a quella delineata dal codice di rito, che però comunque richiede alla parte di individuare i documenti di cui chiede al giudice di ordinare l'esibizione (una volta che "abbia fornito seri indizi della fondatezza delle proprie domande e abbia individuato documenti, elementi o informazioni detenuti dalla controparte che confermino tali indizi", allora la parte "può ottenere che il giudice ne disponga l'esibizione oppure che richieda le informazioni alla controparte").

Piuttosto, è interessante, ai fini del nostro discorso, vedere la lettura dell'istituto che viene data dalla giurisprudenza. "La discovery - afferma il Tribunale di Roma ${ }^{62}$ - è un mezzo istruttorio che deroga in parte ai principi generali sia di piena disponibilità della prova ad opera delle parti, introducendo con l'ordine del giudice degli elementi di ufficiosità, sia di allocazione dell'onere della prova, ponendolo a carico della parte attrice soltanto quanto agli elementi di prova (seri indizi) e non alla prova piena, il cui onere è spostato sulla controparte a cui i fatti da provare sono più prossimi, con ciò attenuando, senza capovolgerlo, l'onere probatorio della parte attrice sui fatti costitutivi della sua domanda".

\footnotetext{
${ }^{59}$ Diverso è invece l'approccio - maggiormente nella direzione della disclosure inglese - adottato dalla direttiva europea n. 104/2014 (che dovrà essere attuata dal nostro legislatore entro il dicembre del 2016), volta a favorire la proposizione di azioni per il risarcimento del danno causato dalla violazione del diritto della concorrenza. La direttiva, dopo aver considerato l'importanza della prova in questa tipologia di cause e la difficoltà per l'attore di provare, dato che gli elementi di convincimento sono "spesso detenuti esclusivamente dalla controparte o da terzi e non sono sufficientemente noti o accessibili all'attore" (così il considerandum 14), ha ritenuto "opportuno garantire agli attori il diritto di ottenere la divulgazione delle prove rilevanti per la loro richiesta, senza che sia necessario, da parte loro, specificarne i singoli elementi" [corsivo nostro]. Così, l'art. 5 della direttiva dispone che "gli stati membri provvedono affinché, nei procedimenti relativi a un'azione per il risarcimento del danno nell'unione, su istanza di un attore che abbia presentato una richiesta motivata comprendente fatti e prove ragionevolmente disponibili che siano sufficienti a sostenere la plausibilità della sua domanda di risarcimento del danno, i giudici nazionali possano ordinare al convenuto o a un terzo la divulgazione delle prove rilevanti che rientrino nel controllo di tale soggetto, alle condizioni precisate nel presente capo".

${ }^{60}$ D.lgs. 10 febbraio 2005, n. 30.

${ }^{61}$ Ai sensi del comma 1 dell'art. 121, "l'onere di provare la nullità o la decadenza del titolo di proprietà industriale incombe in ogni caso a chi impugna il titolo; salvo il disposto dell'articolo 67 l'onere di provare la contraffazione incombe al titolare". Per un commento cfr. Vanz, Onere della prova e vicinanza della prova delle liti IP, in Giussani (a cura di), Il processo industriale, Torino, 2012, 174 ss.

${ }^{62}$ Trib. Roma , sez. proprietà industriale e intellettuale, 17 gennaio 2012, in Riv. dir. ind., 2013, II, 274.
} 
Revista Eletrônica de Direito Processual - REDP. Volume 16. Julho a dezembro de 2015

Periódico Semestral da Pós-Graduação Stricto Sensu em Direito Processual da UERJ

Patrono: José Carlos Barbosa Moreira. ISSN 1982-7636. pp. 93-111

http://www.e-publicacoes.uerj.br/index.php/redp/index

Dalla presenza di un potere istruttorio del giudice - peraltro subordinato a un presupposto tipico per la concessione di una misura cautelare ${ }^{63}$ - il Tribunale ricava la vigenza di una regola dell'onere della dimostrazione imperniata sul criterio della vicinanza della prova: l'attore avrebbe così l'onere di fornire semplici "indizi" di conferma della propria pretesa, mentre la prova, e il rischio della sua mancanza, spetterebbe al convenuto che è la parte più prossima alla stessa.

È facile obiettare che la giurisprudenza, probabilmente sviata dalla rubrica dell'art. 121, confonde e sovrappone i due piani dell'onere della prova quale regola probatoria e quale regola decisoria e che dalla presenza di un potere istruttorio del giudice (per dipiù subordinato all'istanza di parte) non può essere ricavata una regola della ripartizione del rischio della mancata prova differente da quella dell'art. 2697.

In realtà, a me pare che il ragionamento della giurisprudenza - ponendo in stretta correlazione (con un ragionamento simile a quello che abbiamo visto sviluppato in relazione al processo amministrativo ${ }^{64}$ ) regola probatoria e regola di giudizio - dimostri che la ripartizione del rischio non può prescindere dall'effettiva disponibilità degli elementi di prova e che quindi un ordinamento che non garantisca l'effettivo accesso alla prova della parte non possa poi imporle di soccombere perché non ha rappresentato i fatti da lei allegati.

7. - La concentrazione dell'attenzione sul criterio della vicinanza della prova, pertanto, a mio avviso testimonia l'insofferenza della nostra giurisprudenza verso il sistema di accesso alla prova delineato dal nostro codice che, ancorato a una visione di uguaglianza formale delle parti rispetto ai temi da provare, appare del tutto inidoneo a garantire il diritto delle stesse all'accertamento del diritto vantato.

Il ricorso al criterio, infatti, è inquadrato dalla giurisprudenza nel diritto alla prova quale espressione del diritto di azione: il principio della vicinanza o disponibilità dei mezzi di prova è

\footnotetext{
${ }^{63}$ È critico al riguardo Dondi, Il diritto di esibizione - Struttura e singolarità della esibizione-discovery nelle controversie in materia di proprietà industriale, in Giussani (a cura di), Il processo industriale, cit., 237 ss. ${ }^{64}$ Supra, il precedente paragrafo.
} 
Revista Eletrônica de Direito Processual - REDP. Volume 16. Julho a dezembro de 2015

Periódico Semestral da Pós-Graduação Stricto Sensu em Direito Processual da UERJ

Patrono: José Carlos Barbosa Moreira. ISSN 1982-7636. pp. 93-111

http://www.e-publicacoes.uerj.br/index.php/redp/index

"riconducibile all'art. 24 Cost. e al divieto di interpretare la legge in modo da rendere impossibile o troppo difficile l'esercizio dell'azione in giudizio" ${ }^{65}$.

Nell'impossibilità di conferire alla parte che allega il fatto, ma non ha la disponibilità degli elementi indispensabili per rappresentarlo, gli strumenti per ottenere tali elementi, la giurisprudenza agisce onerando la parte che non fornisce la prova di cui dispone del rischio circa il fatto allegato dalla controparte, esercitando così una pressione psicologica nei suoi confronti a collaborare all'istruzione probatoria.

La tecnica utilizzata dai giudici è stata oggetto di critiche severe in dottrina. Si sottolinea da un lato l'eccessiva discrezionalità che il giudice viene così ad attribuirsi ${ }^{66}$ e dall'altro lato che se la giustificazione di acquisizione degli elementi di prova può apparire epistemicamente valida, lo scopo sarebbe perseguito in modo errato e eccessivo, in quanto sarebbe sufficiente prevedere un generale obbligo di disclosure per le parti del processo ${ }^{67}$.

La seconda critica non convince: è indubbio che l'accesso alla prova verrebbe meglio garantito da un sistema probatorio che imponesse alle parti di comunicare tutti gli elementi rilevanti per la prova dei fatti del processo piuttosto che da una regola di giudizio che le stimoli a farlo, ma appunto occorre che tale obbligo di disclosure sia imposto dal legislatore e, al momento, non pare che questa sia la prospettiva del nostro ordinamento.

Quanto alla prima critica, è innegabile che l'applicazione del criterio - come abbiamo visto sottolinea la dottrina spagnola - comporta il pericolo per la parte di una decisione discrezionalmente assunta del giudice. D'altro canto è illusorio, come abbiamo già detto supra ${ }^{68}$, pensare che l'applicazione dell'art. 2697 avvenga in modo meccanico, senza contare che un

\footnotetext{
${ }^{65}$ Così Cass., 25 luglio 2008, n. 20484, già menzionata (negli stessi termini numerose altre pronunzie della Suprema Corte, come, da ultimo, Cass., 9 agosto 2013, n. 19146, circa la ripartizione dell'onere tra appaltante e committente in relazione all'azione di garanzia per le difformità e i vizi dell'opera); l'inquadramento del criterio della vicinanza alla prova all'interno dell'esercizio del diritto di difesa si deve, per la prima volta, alla già più volte ricordata pronunzia delle sezioni unite n. 13533/2001.

${ }^{66}$ Di dottrina delle corti parla Busnelli, La dottrina delle Corti e il risarcimento del danno alla persona, in Danno e responsabilità, 2014, $466 \mathrm{~s}$.

${ }^{67}$ Il riferimento è soprattutto a Taruffo, di cui si veda, tra i numerosi interventi sul tema delle c.d. presunzioni giurisprudenziali, La semplice verità, cit., 232 ss.

${ }^{68}$ Cfr. il paragrafo 1.
} 
Revista Eletrônica de Direito Processual - REDP. Volume 16. Julho a dezembro de 2015

Periódico Semestral da Pós-Graduação Stricto Sensu em Direito Processual da UERJ

Patrono: José Carlos Barbosa Moreira. ISSN 1982-7636. pp. 93-111

http://www.e-publicacoes.uerj.br/index.php/redp/index

potere discrezionale circa l'allocazione del rischio della prova è conferito al giudice dallo stesso legislatore con la previsione dell'istituto del giuramento suppletorio ${ }^{69}$.

Certo, vi è la possibilità che la parte trovi applicato a proprio sfavore il criterio della vicinanza della prova "a sorpresa" in sede di decisione della causa. Occorre quindi - come è previsto dal nuovo codice di rito brasiliano - che la questione della disponibilità della prova venga sottoposta al contraddittorio delle parti, così che queste, e in particolare quella onerata, possano collaborare all'istruttoria avendo chiare le conseguenze in punto rischio del mancato convincimento del giudice ${ }^{70}$.

D'altro canto già Bentham sottolineava che l'individuazione della parte che più facilmente può provare I fatti del processo deve avvenire - secondo la procedura naturale - al primo incontro tra le parti e il giudice ${ }^{71}$.

Mi pare quindi di poter concludere che, grazie all'orientamento della giurisprudenza, nell'ordinamento italiano stia prendendo forma un sistema probatorio basato sul principio di collaborazione tra le parti e il giudice, principio che, codificato in altri ordinamenti (in

\footnotetext{
${ }^{69}$ Conferendo al giudice il potere di scegliere la parte cui deferire il giuramento, il legislatore gli consente di non applicare, a sua discrezione, la regola di giudizio e così di mutare la situazione delle parti rispetto alla prova (l'osservazione è di Benvenuti, L'istruzione nel processo amministrativo, 449, nota 159; sul punto si veda già Pescatore, La logica del diritto, cit., 196 ss.).

${ }^{70}$ In tal senso Taruffo, La semplice verità, cit., 237, il quale però nel successivo lavoro L'onere come figura processuale (in Riv. trim. dir. proc. civ., 2012, 425, nota 22) solleva dubbi sul fatto che la nuova formulazione dell'art. 101 c.p.c., cha fa riferimento alle sole questioni rilevabili d'ufficio, sia strumento idoneo a risolvere il problema, dato che la manipolazione degli oneri probatori non è questione in senso tecnico. In effetti la giurisprudenza afferma che la necessità che le questioni evidenziate per la prima volta in sede di decisione debbano essere segnalate dal decidente alle parti, affinché i difensori interloquiscano sul punto, non ha "nulla a che vedere con l'applicazione del principio per cui il giudice può e deve accogliere la domanda solo quando ritenga dimostrati i fatti costitutivi del diritto azionato e, reciprocamente indimostrate le eccezioni volte a far valere l'inefficacia di tali fatti ovvero che il diritto si è modificato o estinto" (così Cass., 6 novembre 2013, n. 24861). La posizione espressa dalla Corte, però, mi trova solo parzialmente d'accordo: se è vero che l'applicazione dell'art. 2697 non è questione che "fonda" la decisione è però questione che tale decisione orienta, così che l'individuazione della parte che è più vicina alla prova mi pare tema che - ai sensi dell'art. 183, comma 4, c.p.c. - deve essere sottoposto al contraddittorio delle parti.

${ }^{71}$ Bentham, An Introductory View of the Rationale of Evidence, cit., $§ 1$.
} 
Revista Eletrônica de Direito Processual - REDP. Volume 16. Julho a dezembro de 2015

Periódico Semestral da Pós-Graduação Stricto Sensu em Direito Processual da UERJ

Patrono: José Carlos Barbosa Moreira. ISSN 1982-7636. pp. 93-111

http://www.e-publicacoes.uerj.br/index.php/redp/index

particolare, in quello francese ${ }^{72}$ ), in Italia aveva ricevuto attenzione e rilievo da un settore, minoritario e non recente, della dottrina ${ }^{73}$.

Prendendo a prestito le parole di un giurista francese, l'indirizzo espresso dai giudici con il criterio della vicinanza della prova ci dice che "non è più il tempo ove una visione individualista del processo civile portava a pensare che la determinazione dell'onere della prova fissasse a priori il ruolo di ciascuna parte nella ricerca degli elementi per il convincimento del giudice e dove il convenuto beneficiava di una sorta di diritto alla passività: oggi, ciascuno deve portare la sua pietra per l'edificio probatorio"74.

\footnotetext{
${ }^{72}$ Dopo aver prescritto il potere del giudice di ordinare d'ufficio tutte le misure istruttorie ammesse dalla legge (art. 10 c.p.c.: «le juge a le pouvoir d'ordonner d'office toutes les mesures d'instruction légalement admissibles »), il codice di rito francese prevede infatti, all'art. 11, che le parti sono tenute a dare il loro contributo alle misure istruttorie - contributo che, afferma la Cour de cassation, va dato da ciascuna parte, sia quella cui l'onere della prova spetta che quella che dall'onere è dispensata (Cass., 30 marzo 2005, n. 02.20.429, in Procédures, Mai 2005, $14 \mathrm{~s}$.) - e il giudice trae le conseguenze dell'eventuale loro astensione o rifiuto di collaborare. Si è espressa nel senso dell'impossibilità, oggi, di applicare in modo automatico la regola dell'onere della prova la Cour de cassation, con la sentenza 9 gennaio 2014, n. 12.25-472, in Procédures, marzo 2014, 10 s. Per letture dell'onere della prova quale onere di collaborazione delle parti all'istruzione probatoria si vedano Bergeaud, Le droit à la preuve, Paris, 2010, e Le Bars, De la théorie des charges de la preuve et de l'allégation à la théorie globale des risques processuels, in Mélanges Gilles Goubeaux, Paris, 2009, 319 ss.

${ }^{73}$ Fondamentale, per l'affermazione e l'analisi del principio di collaborazione nel nostro ordinamento, resta infatti il contributo di Grasso, La collaborazione nel processo civile, in Riv. dir. proc., 1966, 580 ss.

${ }^{74}$ Perrot, nel commento a Cour de cassation, 9 gennaio 2014, n. 12.25-472, sopra citata.
} 